\title{
Fetal Warfarin Syndrome
}

National Cancer Institute

\section{Source}

National Cancer Institute. Fetal Warfarin Syndrome. NCI Thesaurus. Code C98906.

A teratogenic disorder observed in a newborn or child of a mother who was exposed to warfarin during pregnancy. Manifestations include nasal bridge depression, nasal bones hypoplasia, microcephaly, congenital heart disorders, and brachydactyly. 